\title{
10 Do inequality measures measure inequality?
}

Gary Fields

\section{Introduction}

I first met Aldi Hagenaars at the World Econometric Society Congress in Cambridge, Massachusetts in 1985. At that time, we had a long discussion about the question posed in the title of this chapter. The driving force behind Aldi's research program became clear to me then: a profound concern with people's feelings about their economic well-being accompanied by the quest to devise measures that reflect those underlying feelings.

This chapter follows that tradition. My own research program has dealt with the twin questions of who benefits how much from economic growth and why. Before answering these questions, it is necessary to decide what measure to take to the data to determine if economic growth is welfare-enhancing. For many people, one component of a social welfare judgment is the extent of income inequality.

There is no shortage of inequality measures (Gini coefficient, Theil index, Atkinson index, etc.). The task is to decide what we mean by "inequality" and then to determine which, if any, of the available measures behave as "inequality" does.

The literature offers two principal ways of relating social welfare to the inequality of income. ${ }^{1}$ One, due to Kolm (1966) and Atkinson (1970), first constructs a social welfare function defined on the space of incomes

$$
W=W\left(y_{1}, y_{2}, \ldots, y_{n}\right)
$$

then defines the equally distributed income as the amount of income which, if equally distributed would yield the same social welfare as the actual distribution, and finally measures inequality as the gap between the actual mean income $\bar{y}$ and the equally distributed income $y^{*}$ 


$$
I=I-\left(y^{*} / \bar{y}\right)
$$

Another way of taking the level of national income and the inequality of its distribution into account in welfare judgments is to rank income distributions in terms of the mean income level $\bar{Y}$, income inequality $I$ and perhaps other things:

$$
W=f(\bar{Y}, I, \ldots), \partial W / \partial \bar{Y}>0, \partial W / \partial I<0
$$

Ranking income distributions in terms of income level and income inequality is common practice in a whole host of empirical studies of "growth and equity." This practice receives abundant theoretical support, for instance, in Sen (1973), Blackorby and Donaldson (1977, 1984), Fields (1979), Ebert (1987), and Lambert (1989). Schur-concave social welfare functions provide one justification for this practice; another is diminishing marginal utility of identical interpersonally comparable utility functions.

To be able to rank income distributions in this way, we must determine how the inequality of one distribution compares to that of another, which means that the primitive concept of "inequality" must be made precise. Amartya Sen (1973, pp. 2, 3) has written:

there are some advantages in . . . try[ing] to catch the extent of inequality in some objective sense ... so that one can distinguish between (a) "seeing" more or less inequality, and (b) "valuing" it more or less in ethical terms ... There is, obviously, an objective element in this notion: a fifty-fifty division of a cake between two persons is clearly more equal in some straightforward sense than giving all to one and none to the other. [First emphasis Sen's, second and third emphases mine.]

It is precisely this objective sense of "seeing" what inequality is which I shall adopt in this chapter in the context of inequality comparisons.

In the literature, much attention has been paid to a number of aspects of inequality including the distinction between relative and absolute inequality, axiomatization of inequality, the Lorenz criterion for inequality comparisons, properties of various inequality measures, and inequality decomposition. In no way do I wish to argue with the main results derived in these areas. Rather, my purpose here is to add to the theory of inequality measurement by dealing with one aspect of inequality which has been largely ignored by economists ${ }^{2}$ and by others. ${ }^{3}$ This is the question of how inequality changes - in particular, whether it increases, decreases, or remains unchanged - when income grows in specified ways. ${ }^{4}$

The balance of this chapter deals with two distinct conceptual entities, "inequality" and "inequality measures." The next section analyzes how 
"inequality" might be said to change under various types of economic growth and explores the foundations for alternative views. One approach in terms of "elitism of the rich" and "isolation of the poor" is then described. The following section looks into the behavior of "inequality measures" and the relationship between "inequality measures" and "inequality," and a final section draws some conclusions.

\section{How does inequality change with economic growth?}

In this chapter, inequality is analyzed on the space of "incomes" among "persons," ruling out multiple goods and problems of aggregation. The analysis proceeds axiomatically, following a long tradition which dates back at least to Pigou (1912) and Dalton (1920) and has been accepted by many others ever since. ${ }^{5}$ It holds that whenever a transfer of income is made from a person ${ }^{6}$ who is relatively poor to another who is relatively rich, inequality increases. Notice two things about this way of conceptualizing the primitive concept "inequality." First it is in terms of conditional "if ... then" statements. Second, the answer to the question "what is inequality?" is sought by looking at inequality orderings on pairs of distributions, addressing the related question "when is one distribution more equal than another?"' I shall follow this practice and seek to clarify the meaning of "inequality" by formulating a series of conditional statements on binary comparisons.

Let the economy consist of $n$ "persons," total population being assumed fixed. Let $\Phi$ be the share of persons having income $y_{H}$ and $1-\boldsymbol{\Phi}$ the share having income $y_{L}\left(<y_{H}\right)$. Analyzing this restricted domain is helpful in forming precise views on the meaning of inequality before moving on to analyze inequality on the more general domain of incomes, a task left for the future.

Throughout the rest of this chapter, we shall use the notation $\Phi=n H / n$ and $\Theta=Y_{H} / Y_{L}$ whenever $\Phi \in(0,1) .{ }^{7}$ The term "increase in $\Theta$ " shall be understood as signifying an increase in $Y_{H} / Y_{L} \forall \Phi \in(0,1)$.

In the two-income world, income growth can take place by increasing $Y_{H}, Y_{L}$, or $\boldsymbol{\Phi}$, or by some combination of these. A simple example shows why inequality rankings are sometimes difficult to make when incomes are growing, even in so simple a world.

Consider an economy consisting of six individuals with an initial distribution of income $[1,1,1,1,1,6]$. Now suppose the economy experiences income growth of $\$ 5$. The change in inequality depends on how that $\$ 5$ is distributed.

First let the entire $\$ 5$ go to the rich person. We have little difficulty in ranking the new distribution $[1,1,1,1,1,11]$ as more unequal than the old. 
Suppose instead that the $\$ 5$ of income growth is divided equally among all the low income persons, resulting in a new distribution $[2,2,2,2,2,6]$. Once again, the ranking is likely to be uncontroversial: the new distribution may be said to be more equal than the old.

Consider a third possibility: that the $\$ 5$ of income growth produces an income gain for just one of the low income persons. ${ }^{8}$ Compare the two income distributions

$$
Y_{1}=[1,1,1,1,1,6]
$$

and

$$
Y_{2}=[1,1,1,1,6,6]
$$

Which is more equal, $Y_{1}$ or $Y_{2} ?^{9}$ The rest of this chapter seeks an answer.

A natural starting point would be Lorenz curve comparisons. One income distribution is said to Lorenz-dominate another if the first distribution's Lorenz curve is somewhere above and nowhere below that of the second. The Lorenz criteria for relating the inequality of two distributions consist of several parts:

(i) If two distributions $X, Y \in Z$ have the same Lorenz curves $L_{x}$ and $L_{y}$, then they are equally unequal $\left(=_{L}\right)$ by the Lorenz criterion, i.e.

$$
L_{X}=L_{Y} \Rightarrow X={ }_{L} Y
$$

(ii) If distribution $X$ Lorenz dominates distribution $Y$, then $X$ is more equal than $Y$ by the Lorenz criterion $(L)$ :

$$
L_{X} \succeq L_{Y} \Rightarrow X \succ_{L} Y
$$

(iii) If the Lorenz curves of $X$ and $Y$ cross, then the inequality of the two distributions cannot be compared using the Lorenz criterion alone.

Together, these are termed ranking $\succeq_{L}{ }^{10}$

When economic growth causes the income distribution to change from $Y_{1}=[1,1,1,1,1,6]$ to $Y_{2}=[1,1,1,1,6,6]$, we may calculate the cumulative income shares Cum $Y_{1}=[1 / 11,2 / 11,3 / 11,4 / 11,5 / 11,1]$ and Cum $Y_{2}=[1 / 16,2 / 16,3 / 16,4 / 16,8 / 16,1]$ and note that their Lorenz curves cross. Therefore the two distributions' relative inequalities cannot be ranked on the basis of the Lorenz properties alone. Likewise, in a sequence of income distributions whereby an economy progresses from $Y_{1}=[1,1,1,1,1,6]$ to $Y_{2}=[1,1,1,1,6,6]$ to $Y_{3}=[1,1,1,6,6,6]$ to $Y_{4}=[1,1,6,6,6,6]$ to $Y_{5}=[1,6,6,6,6,6]$, all of the associated Lorenz curves cross each other. If we want to say how the inequalities of the different distributions compare, we must go beyond the Lorenz criteria considered thus far and appeal to additional properties. 
One way of sharpening our views about what inequality is is to look at the sequence of distributions from $Y_{1}$ through $Y_{5}$ and see what we "see." 11 From simple eyeballing of the sequence $Y_{1}$ through $Y_{5}$, many different patterns emerge as plausible possibilities. It might be said that inequality decreases monotonically, increases monotonically, follows a U-shaped path, follows an inverted U-shaped path, or even remains unchanged. This does not exhaust the possible patterns.

Experimental evidence confirms the diversity of views for a similar thought experiment. Amiel and Cowell (1992) asked more than 1,000 university students to compare the inequality of two distributions

$$
A=[5,5,5,10]
$$

and

$$
B=[5,5,10,10]
$$

40 percent judged $A$ as more unequal, 56 percent judged $B$ as more unequal, and 4 percent judged them equally unequal. When asked to compare

$$
B=[5,5,10,10]
$$

with

$$
C=[5,10,10,10]
$$

57 percent judged $B$ as more unequal, 40 percent judged $C$ as more unequal, and 3 percent judged them equally unequal. Furthermore, in three-way comparisons, by far the most common judgment was to deem $[5,5,10,10]$ as less unequal than both $[5,5,5,10]$ and $[5,10,10,10]$, a view consistent with the U-shaped pattern.

In addition, Amiel and Cowell's respondents were asked about their views on what I have here called the high income sector-enlargement process. Specifically, the question asked was:

Suppose there is a society consisting of $\mathrm{n}$ people. There is one rich person and $\mathrm{n}-1$ identical poor people. One by one, some of those who were poor acquire the same income as the rich person, so that eventually there are $\mathrm{n}-1$ (identical) rich people and just one poor person. Please circle the appropriate response:

Inequality increases continuously.

Inequality decreases continuously.

Inequality at first increases and then decreases.

Inequality at first decreases and then increases. 
Inequality remains the same throughout.

None of the above.

(The frequency distribution of valid responses is given in brackets).

Here, as well, the U-shaped pattern is the most common.

At this point, I would invite the reader to consider his or her own rankings of the inequality of $Y_{1}$ versus $Y_{2}$ versus $Y_{3}$ versus $Y_{4}$ versus $Y_{5}$, of $A$ versus $B$ versus $C$, and of the high income sector-enlargement process described in the previous paragraph.

\section{Justifying alternative patterns in terms of "elitism of the rich" and "isolation of the poor"}

If you had trouble making the inequality comparisons asked for above, it may be because you lack a framework for moving beyond Lorenz comparisons. This section may help in that regard.

A philosopher, Temkin (1986), has analyzed essentially the same process, different only in that he has total income falling rather than rising. Temkin's analysis is in terms of individual complaints - how serious the inequality in a situation is from the standpoint of particular individuals in that situation. Alternative principles (additive, weighted additive, and maximin) and referents (relative to the average, relative to the best-off person, and relative to all those better off) lead plausibly, in his view, to most of the various patterns raised above.

In an earlier paper (Fields, 1987), I adopted a different approach, which I also take here. Rather than weighing "individual complaints," I look at the question from a societal point of view. The specific way in which I now prefer to do this is with reference to two concepts - "elitism of the rich" and "isolation of the poor" - which are developed formally in Fields (1993).

Briefly put, elitism of the rich (ER) is the following idea. When one person has a high income $\left(\$ y_{H}\right)$ and everybody else has a low income $\left(\$ y_{L}\right.$ each), the one rich person may be thought to have a very elite position. In this case the economy may be said to have a high degree of elitism of the rich. Now let a second person acquire a high income, all others' incomes remaining the same ( $\Phi$ increases). Because each of the rich now has to share his elitist position with someone else, the two rich persons together might be regarded as less elite than one person was when he alone was rich. Thus, elitism of the rich falls. If a third person is enriched, elitism of the rich might be thought to fall further, but not by 


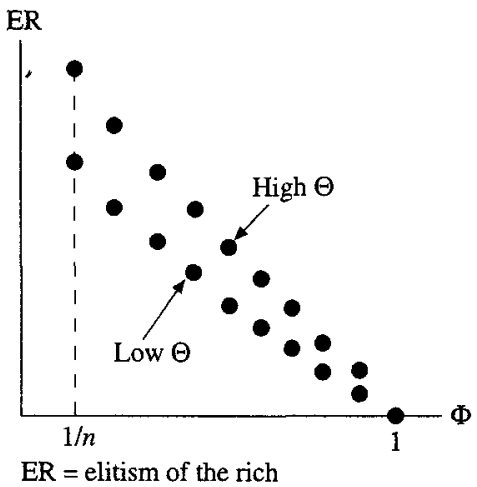

Figure 10.1 Elitism of the rich

as much as when the second person was enriched. In general, the larger the fraction rich, the smaller is elitism of the rich and the smaller is the change in elitism of the rich for a given increase in size of the high income group. When everybody is rich, there no longer is any elitism of the rich.

Elitism of the rich also varies with the ratio of high incomes to low incomes $(\Theta)$. Holding the number of persons in the two income groups constant, if the amount received by each high income person increases or if the amount received by each low income person decreases, elitism of the rich should increase.

Figure 10.1 summarizes how elitism of the rich varies with $\Phi$ and $\Theta$.

Isolation of the poor (IP) may be defined as a reciprocal notion to elitism of the rich. When everyone in an economy is equally poor, there is no isolation of the poor, because there are no rich from whom to be isolated. When one person escapes poverty, isolation of the poor is created. As the number with high incomes increases, those who are left behind may be regarded, as a group, as increasingly isolated. For this reason, isolation of the poor may be viewed as increasing at an increasing rate as the high income group expands. Finally, when just one person is poor, it can be argued that that one person is very isolated from everyone else, at which point isolation of the poor attains a maximum. Now, holding the numbers in the two groups constant any increase in the ratio between high and low incomes $(\Theta)$ should increase isolation of the poor. These properties of isolation of the poor are shown in Figure 10.2.

From these concepts of elitism of the rich and isolation of the poor, we may derive various inequality patterns. Those observers who wish to view inequality solely in terms of elitism of the rich would see inequality 


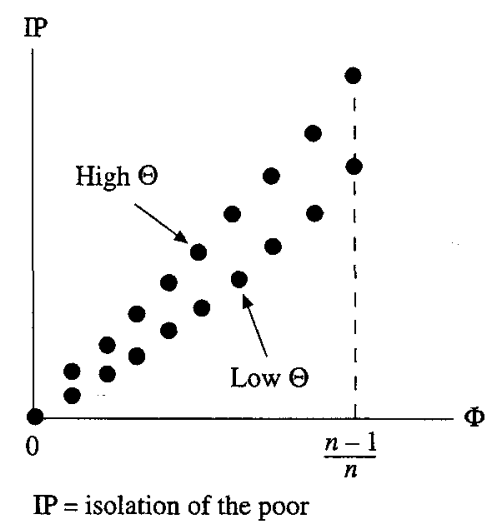

Figure 10.2 Isolation of the poor

as falling continuously on the interval $\Phi \in(0,1)$ for any given $\Theta$. The higher is $\Theta$, the higher is inequality. This class, shown in figure 10.3 , will be termed the $I$ class.

Others may wish to view inequality solely in terms of isolation of the poor. These observers would see inequality as rising continuously on the interval $\Phi \in(0,1)$ for any given $\Theta$. A higher $\Theta$ implies more inequality. Figure 10.4 depicts this class, denoted the $I_{+}$class.

Many observers hold the view that inequality consists of both elitism of the rich and isolation of the poor. How might these notions be combined on their common domain, the open interval $(0,1)$ ?

Elitism of the rich and isolation of the poor need to be expressed in comparable units, which may be done by postulating that for any $\boldsymbol{\Phi}, E R(\Phi)=I P(1-\Phi)$.

Next, elitism of the rich and isolation of the poor need to be combined in some plausible way. Suppose equal weight is given to each. The simplest such mixing function, defined on the open interval $(0,1)$, the common domain of $E R(\cdot)$ and $I P(\cdot)$, is

$$
I(\boldsymbol{\Phi}, \boldsymbol{\Theta})=(E R+I P) / 2
$$

Alternatively, unequal weights may be posited by using a linear mixing function:

$$
I(\cdot)=w E R(\cdot)+(1-w) I P(\cdot), w>0, w \neq 1-w
$$

Define the $I_{\min }$ class to be those $I(\cdot)$ rankings which are U-shaped as $\Phi$ varies on the open interval $(0,1)$ for a given $\Theta$, and which lie on higher contours for higher $\Theta$, as shown in figure 10.5; denote those which also are symmetric with a unique minimum at $\Theta=1 / 2$ as the symmetric $I_{\text {min }}$ 


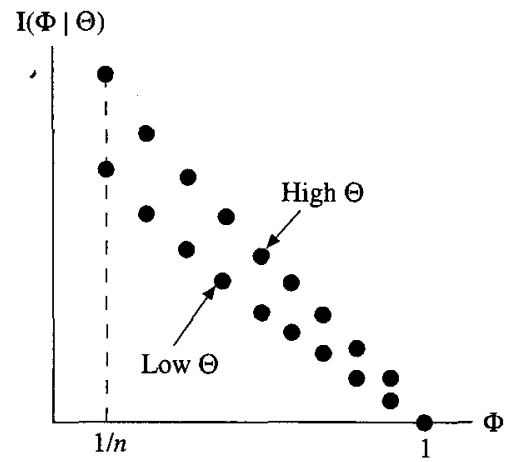

Figure 10.3 The $I_{\text {_ }}$ class of inequality rankings

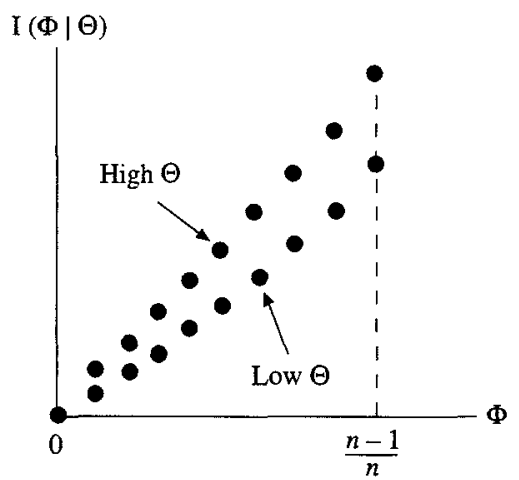

Figure 10.4 The $I_{+}$class of inequality rankings

class. Fields (1993) proves that the preceding properties with equal weights generate the symmetric $I_{\min }$ class and these properties with unequal weights generate a ranking which is a member of the $I_{\min }$ class but not the symmetric $I_{\min }$ class.

Note two things. First, given $I(\cdot)=w E R(\cdot)+(1+w) I P(\cdot)$, for $w=1$, we have the $I_{-}$pattern shown in figure 10.3 , and for $w=0$, the $I_{+}$ pattern shown in figure 10.4, which may be attractive to some observers. Second, these are the only patterns consistent with the preceding axioms. In particular, the inverted-U pattern cannot be generated from the preceding properties.

It remains to extend the inequality ordering to the end-points $\mathbf{\Phi}=0$ and $\boldsymbol{\Phi}=1$. If we adopt the Lorenz axioms, we obtain two additional restrictions on the inequality orderings. One restriction, arising from the transfer principle, is that a situation in which everyone has the same income must be regarded as more equal than any situation in which 


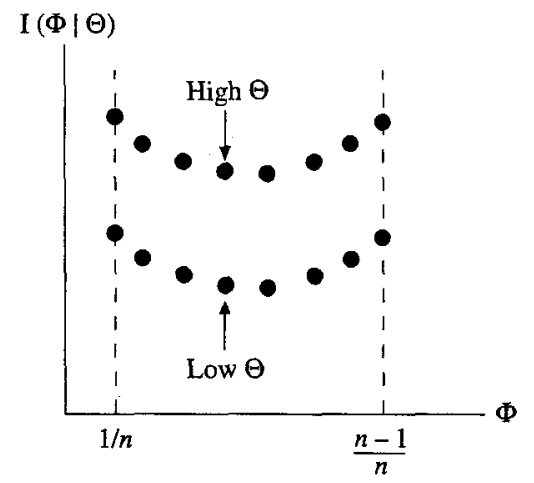

Figure 10.5 The $I_{\min }$ class of inequality rankings

incomes differ. Accordingly, the Lorenz properties require that a situation of equally distributed incomes be deemed a "most equal point." The other restriction, arising from the income homogeneity principle, is that all equally distributed incomes be deemed "most equal points." If we adopt a natural normalization - that most equal points have no inequality - we then have

$$
I(Y / n, Y / n, \ldots Y / n)=0
$$

for all total income amounts $Y$.

The concept of a most equal point accords with the notions introduced earlier of elitism of the rich and isolation of the poor. Suppose that two incomes are possible but that everybody has one of those incomes and nobody has the other. In one case, there is no elitism of the rich, because there are no rich; and in the other case, there is no

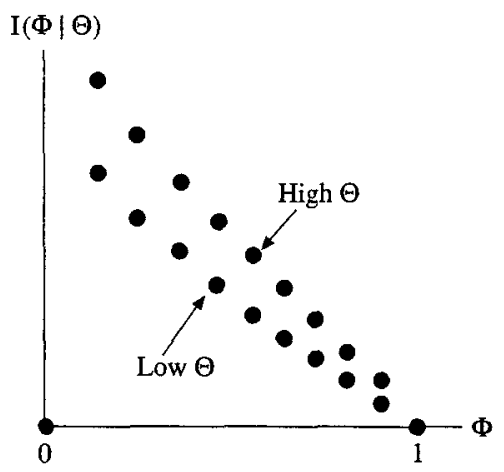

(a) $I_{-}^{L}$ 
Do inequality measures measure inequality?

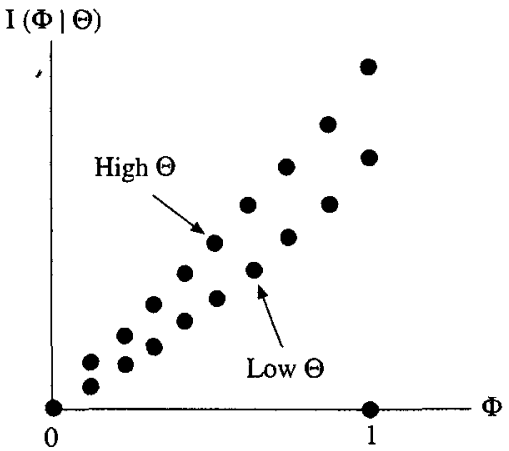

(b) $I_{+}^{L}$

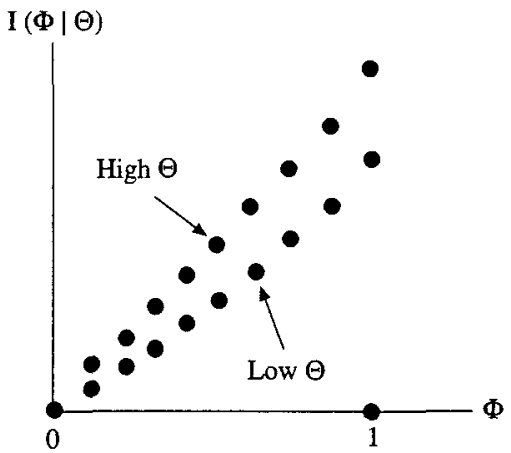

(c) $I_{\min }^{L}$

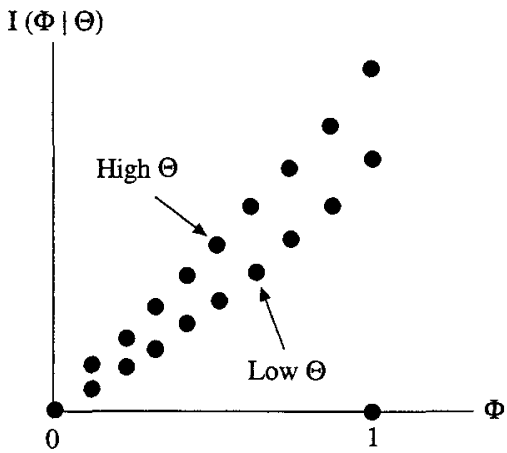

(d) $I_{\max }^{L}$

Figure 10.6 The $I^{L}$ classes of inequality rankings 
isolation of the poor, because there are no poor. One would be inclined to say that a situation where all are equally rich or equally poor is more equal than a situation where some are rich and some are poor and that the two situations ("all rich" and "all poor") have the same inequality as one another. Inequality notions in the $I_{-}^{L}, I_{+}^{L}$, and $I_{\min }^{L}$ classes make these judgments.

Combining these judgments with the alternative enlargement patterns as depicted in figures 10.3 through 10.5 , we obtain the three inequality orderings $I_{-}^{L}, I_{+}^{L}$, and $I_{\text {min }}^{L}$ shown in figures $10.6 \mathrm{a}-6 \mathrm{c}$ respectively. All are Lorenz consistent, yet they treat high income sector enlargement in very different ways.

Note, too, that the familiar inverted U-shaped pattern shown in figure $10.6 \mathrm{~d}$ cannot be generated from the preceding axioms. A different justification is needed. I shall not pursue that here.

\section{Inequality measures and inequality}

Any relative inequality relation such as those considered on p. 236 determines which of two income vectors $X, Y \in Z$ is more equal than the other. An inequality function $I(\cdot)$ which assigns a real number $r$ to $X$ and $Y$ representing the inequality of that vector such that $I(X)$ and $I(Y)$ are ordered by the usual greater than or equal to relation $\geq$ is said to be an inequality measure (alternatively, a "numerical inequality measure" or an "inequality index"). A relative inequality measure has the additional property that an equiproportionate change in everyone's income leaves inequality unchanged.

Some relative inequality measures are Lorenz consistent (meaning that they make precisely those judgments specified by the Lorenz properties whenever Lorenz comparisons can be made) and some are not, either because they are only weakly Lorenz consistent (meaning that if one Lorenz curve lies partly above but not below another, the measure may rank the two distributions as equally unequal) or because they are Lorenz inconsistent (meaning that there may be a case in which one distribution Lorenz dominates another and yet the measure deems the first distribution to be more unequal than the second). Among those known to be Lorenz consistent are the Gini coefficient, Theil's two measures, Atkinson's index, and the coefficient of variation. Among those known not to be Lorenz consistent are some which are only weakly Lorenz consistent (income share of the richest $X \%$ or poorest $Y \%$ ) and others which are Lorenz inconsistent (including the mean absolute deviation and the logarithm of the variance of incomes). Lorenz consistent inequality measures are emphasized hereafter. 
It will be said that an inequality measure measures inequality, or equivalently, that the inequality measure is consistent with a specified ranking, when the ordinal ranking assigned by the inequality measure matches the ordinal ranking of inequality assigned by the observer. All that can be hoped for is agreement on conditional statements of the type: "For all those whose inequality notions are of such and such type, such and such inequality measures measure inequality (and such and such other inequality measures do not)."

Consider now the process of high income sector enlargement on the domain of two incomes, as analyzed on pp. 235-44. It was shown in Fields (1993), building on the work of Anand and Kanbur (1993), that each of six commonly used inequality measures (Theil's entropy index, Theil's second measure, $\mathrm{CV}^{2}$, Atkinson index, Gini coefficient, nonoverlapping case, and log variance) starts at zero, increases continuously to an interior maximum at $\Phi^{*}$, and then decreases to zero. ${ }^{12}$

Thus, Theil's entropy index, Theil's second measure, the $\mathrm{CV}^{2}$, the Atkinson index, and the Gini coefficient (non-overlapping case) are Lorenz consistent and follow an inverted U-shaped pattern in high income sector-enlargement growth. ${ }^{13}$ These commonly used measures therefore go beyond the Lorenz ordering in a way that produces a similar pattern of rankings (the inverted U-pattern). ${ }^{14}$

Turning now to the question of symmetry, it is sometimes said that on the two-income domain, inequality should reach a turning point when half the population is in the high income group and half in the low income group, i.e. at $\Phi^{*}=1 / 2$. Do the five Lorenz consistent inequality measures considered above have this property? It can be shown by numerical example that the answer is "no." Those who believe that inequality should increase until half the population is in the high income group and decrease thereafter might find this result disturbing.

There is, though, an inequality measure that follows the inverted $U$ shaped pattern and does turn at $\Phi=1 / 2$, namely, the log variance. It, however, is not Lorenz consistent.

These five commonly used inequality measures (excluding the log variance, which is not Lorenz consistent) all belong to the same subclass, namely, the $I_{\max }^{L}$ subclass depicted in figure $10.6 \mathrm{~d}$. Is it possible to construct Lorenz consistent inequality measures possessing the $I_{\min }^{L}$ pattern depicted in figure 10.6c? The answer is "yes." An example of such a measure is:

$$
I=(\Theta-1)^{\alpha}(K+1 / 4-\mathbf{\Phi}(1-\boldsymbol{\Phi}))^{1-\alpha}
$$


where

$$
\begin{aligned}
\boldsymbol{\Theta} & =y_{H} / y_{L}, \boldsymbol{\Phi} \in(0,1) \\
& =1, \boldsymbol{\Theta}=0,1 \\
0 & <\alpha<1
\end{aligned}
$$

and

$$
K>0 .{ }^{15}
$$

This index is but one example of a real-valued function with the desired properties. There are many other possible representations, e.g. $I^{\prime}=\left(\Theta^{2}-1\right)^{\alpha}(K+1 / 4-\Phi(1-\Phi))^{1-\alpha}$. It remains to explore the properties of various alternatives and determine their relative merits.

\section{Conclusion: do inequality measures measure inequality?}

This chapter has set out different views which prescribe how inequality rankings "ought" to behave in certain circumstances. The Lorenz axioms have been unchallenged. The contribution of this chapter has been to analyze inequality orderings when Lorenz curves cross.

The findings in this chapter raise two conceptual problems for empirical researchers. One problem confronts those who use these measures in empirical applications but who have not yet decided which enlargement pattern they favor. Use of the standard measures implicitly imposes an ordering. Before using one of these measures, the researcher should ask whether the ordering imposed (the $I_{\max }^{L}$ ordering) is the one he or she wïshes to impose.

A different problem arises for those of us who believe that inequality "should" be highest when most people are in one income group and few are in the other. For such observers, the standard measures do not do what they "should" do.

The most important task for future work is to expand the domain to allow for intra-group inequality, and for more than two groups.

\section{Notes}

This research was carried out at Cornell University, DELTA in Paris, France and at the Suntory-Toyota International Centre for Economics and Related Disciplines, London School of Economics. I am grateful for those institutions' support of this work. I thank Tony Atkinson, François Bourguignon, Frank Cowell, James Foster, Aldi Hagenaars, Bob Hutchens, George Jakubson, Peter Lambert, Pierre Pestieau, Debraj Ray, Amartya Sen, Tony Shorrocks, Nick Stern, Amos Witztum, Manny Yaari, and an anonymous referee for helpful comments and discussion. 
1 Use of the term "income" is purely for verbal convenience. Inequality of consumption or of anything else could be treated identically:

2 But see Fields $(1987,1993)$.

3 But see Temkin (1986).

4 Attention here is limited to the ordinal aspects of the problem.

To avoid possible misunderstanding of the purpose of this chapter, let me state explicitly that it is not about the question of when one distribution is better than another. Possible criteria for such judgments include comparisons of income level and income inequality, the Pareto criterion on the space of incomes, the Rawlsian maximin criterion, comparisons of generalized Lorenz curves, Kolm's criteria for optimal justice, and various dominance criteria. These criteria from welfare economics, as well as others from ethics and political philosophy, are reviewed and developed by Sen (forthcoming). But all of these criteria are for welfare rankings and this chapter is about inequality rankings, on which Sen's earlier work (Sen, 1973) is a classic.

5 See, for instance, Sen (1973), Atkinson (1983), Foster (1985), and the references cited therein.

6 "Person" stands for whatever recipient unit is relevant, which may be an individual, a family, or per capita.

7 When $\Phi=0$ or $1, \Theta=1$.

8 Analysis of this process is of more than academic interest: Simon Kuznets won a Nobel Prize in part for showing that the gradual shift of economic activity from low income to high income sectors is the essence of modern economic growth and for analyzing inequality change under such a process (Kuznets, 1955, 1966).

9 Again please remember that the question is "which is more equal?", not "which is better?" The great majority of observers rank $Y_{2}$ as better than $Y_{1}$.

10 Ranking inequalities using $\succeq_{L}$ is equivalent to making inequality comparisons using four basic properties: anonymity, income homogeneity, population homogeneity, and the Pigou-Dalton transfer principle. For details, see Dasgupta, Sen and Starrett (1973), Rothschild and Stiglitz (1973), and Fields and Fei (1978). Contrary to these relative approaches, Kolm (1976) and Eichhorn and Gehrig (1982) are among those who have adopted more absolute perspectives on inequality comparisons. For a review of the literature on non-relative views, see Ebert (1987).

11 Aldi Hagenaars liked this approach; we had a long, fruitful talk about it.

12 Such a result was already known for the Gini coefficient from the work of Knight (1976) and Fields (1979) and was implied by work on the coefficient of variation by Swamy (1967), on the log variance by Robinson (1976), and on the generalized entropy class, the Atkinson index, and the income share of the poorest $p$ percent by Kakwani (1988).

13 The $\log$ variance, although not Lorenz consistent, also follows an inverted U-shaped pattern.

14 A higher value of Atkinson's inequality aversion parameter $\varepsilon$ produces a smaller value of $\Phi^{*}$. As $\varepsilon \rightarrow \infty$, the Atkinson index produces peak inequality 
at $\boldsymbol{\Phi}=1 / n$, thus representing the $I^{L}$-ordering. I am grateful to Tony Atkinson for pointing this out to me.

$15 L>0$ guarantees that $I>0$ for all $\Phi$, in particular, at the interior minimum. The necessity of including such a parameter was first pointed out to me by Aldi Hagenaars and James Foster.

\section{References}

Amiel, Y. and F. Cowell, 1992. "Measurement of income inequality: experimental test by questionnaire," Journal of Public Economics, 47, 3-26

Anand, S. and R. Kanbur, 1993. "The Kuznets process and the inequalitydevelopment relationship," Journal of Development Economics, 40, 25-52

Atkinson, A.B., 1970. "On the measurement of inequality," Journal of Economic Theory, 2, 244-63

1983. Social Justice and Public Policy, Cambridge, MA: MIT Press

Blackorby, C. and D. Donaldson, 1977. "Utility vs. equity: some plausible quasiorderings," Journal of Public Economics, 7, 365-81

1984. "Ethically significant ordinal indexes of relative inequality," Advances in Econometrics, 3, 131-47

Dalton, H., 1920. "The measurement of the inequality of incomes," Economic Journal, 3, 348-61

Dasgupta, P., A. Sen and D. Starrett, 1973, "Notes on the measurement of inequality," Journal of Economic Theory, 6, 180-7

Ebert, U., 1987. "Size and distribution of incomes as determinants of social welfare," Journal of Economic Theory, 41, 23-33

Eichhorn, W. and W. Gehrig, 1982. "Measurement of inequality in economics," in B. Korte (ed.), Modern Applied Mathematics, Amsterdam: North-Holland

Fields, G.S., 1979. "A welfare economic analysis of growth and distribution in the dual economy," Quarterly Journal of Economics, 93, 325-53

1987. "Measuring inequality change in an economy with income growth," Journal of Development Economics, 26, 357-74

1993. "Inequality in dual economy models," Economic Journal, 103, 1228-1235

Fields, G.S. and J.E.H. Fei, 1978. "On inequality comparisons," Econometrica, $46,303-16$

Foster, J., 1985. "Inequality measurement," in H. Peyton Young (ed.), Fair Allocation, Providence, RI: American Mathematical Society

Kakwani, N., 1988. "Income inequality, welfare and poverty in a developing economy with applications to Sri Lanka," in W. Gaertner and P.K. Pattanaik (eds.), Distributive Justice and Inequality, New York: SpringerVerlag

Knight, J.B., 1976. "Explaining income distribution in less developed countries: a framework and an agenda," Bulletin of the Oxford Institute of Economics and Statistics, 58, 161-77

Kolm, S.C., 1966. "The optimal production of social justice," in H. Guitton and J. Margolis (eds.), Public Economics, London: Macmillan 
1976. "Unequal inequalities," Journal of Economic Theory, 12, 416-42.

Kuznets, S., 1955. "Economic growth and income inequality," American Economic Review, 45, 1-28

1966. Modern Economic Growth, New Haven: Yale University Press

Lambert, P., 1989. The Distribution and Redistribution of Income, Cambridge, MA: Blackwell

Robinson, S., 1976. "A note on the $U$ hypothesis relating income inequality and economic development," American Economic Review, 66, 437-40

Rothschild, M. and J. Stiglitz, 1973. "Equilibrium in competitive insurance markets: an essay on the economics of imperfect information," Quarterly Journal of Economics, 90, 629-50

Sen, A.K., 1973. On Economic Inequality, Oxford: Oxford University Press

1982. Choice, Welfare and Measurement, Oxford: Basil Blackwell (forthcoming). Equality and Diversity

Shorrocks, A. and J. Foster, 1987. "Transfer sensitive inequality measures," Review of Economic Studies, 54, 485-97

Swamy, S., 1967. "Structural changes and the distribution of income by size: the case of India," Review of Income and Wealth, 13, 155-74

Temkin, L.S., 1986. "Inequality," Philosophy and Public Affairs, 15, 99-121 